\title{
Acceso y actitud del uso de Internet entre jóvenes de educación universitaria
}

\author{
Alejandra Morales Ramírez * \\ Universidad Autónoma del Estado de México, Toluca de Lerdo, México \\ http://orcid.org/0000-0002-8737-5985 \\ Jessica Danae Zacatenco Cruz \\ Universidad Autónoma del Estado de México, Toluca de Lerdo, México \\ https://orcid.org/0000-0001-6603-5394 \\ Martín Luna Luna \\ Universidad Autónoma del Estado de México, Toluca de Lerdo, México \\ https://orcid.org/0000-0003-2677-4031 \\ Rodolfo Zolá García Lozano \\ Universidad Autónoma del Estado de México \\ https://orcid.org/0000-0003-1087-6156 \\ Cuauhtémoc Hidalgo Cortés \\ Universidad Autónoma del Estado de México \\ http://orcid.org/0000-0001-6324-7180
}

Recibido:06/12/2018 Revisado: 03/03/2019 Publicado: 14/04/2020

\section{Resumen}

El presente artículo propone identificar la actitud y las prácticas habituales de acceso a Internet entre los universitarios; además de conocer su grado de relación con la edad, género, semestre, carrera y rendimiento académico. Para ello, se utilizó un enfoque cuantitativo y un diseño correlacional a través de un cuestionario sociodemográfico, y la escala Telephone, Tool, Territory, Treasure of Information y Toy (5T). Participaron 813 estudiantes de una universidad pública de México. Los resultados mostraron que, desde hace varios años, el uso de Internet es masivo entre los universitarios, aunque existen diferencias significativas en cuanto al tipo de contenido al que acceden. Los hombres, los participantes inscritos en segundo semestre y los que tienen 21 años puntúan más alto cuando utilizan el Internet para jugar y como entretenimiento en comparación con el resto de sus compañeros. También se demostró que no existe una relación entre los usos de Internet, edad y rendimiento académico. Por último, se discuten las implicaciones de los resultados hallados.

Palabras clave: Internet; acceso; actitud; estudiantes universitarios.

\section{Access and attitude of Internet use among college students}

\footnotetext{
Abstract

This article proposes to identify the attitude and habitual practices of Internet access among university students, in addition to knowing their degree of relationship with age, gender, semester, career and academic performance. For this, a qua-
} 
litative and correlational research model will be used through a sociodemographic questionnaire and the scale Telephone, tool, territory, information treasure and toy (5T). 813 students from a public university in Mexico participated. The use of the Internet is massive among university students, there are specific differences in the type of content they access. Men, participants enrolled in the second semester and those who are 21 years old score higher when they use the Internet to play and as entertainment compared to their peers. There is no relationship between Internet uses, age and academic performance. The implications of the results found are discussed.

Keywords: Internet; access; attitude; college students

\title{
Acesso e atitude do uso da Internet entre jovens do ensino universitário.
}

\begin{abstract}
Resumo
Este artigo propõe identificar a atitude e as práticas habituais de acesso à Internet entre estudantes universitários; Além de conhecer seu grau de relação com idade, sexo, semestre, carreira e desempenho acadêmico. Para tanto, utilizou-se uma abordagem quantitativa e um desenho correlacional, por meio de um questionário sociodemográfico, e a escala Telefone, Ferramenta, Território, Tesouro da Informação e Brinquedo (5T). Participaram 813 estudantes de uma universidade pública do México. Os resultados mostraram que, por vários anos, o uso da Internet é massivo entre estudantes universitários, embora haja diferenças significativas no tipo de conteúdo que eles acessam. Homens, participantes matriculados no segundo semestre e aqueles com 21 anos obtêm uma pontuação maior quando usam a Internet para jogar e como entretenimento em comparação com outros parceiros. Também foi demonstrado que não há relação entre uso da Internet, idade e desempenho acadêmico. Finalmente, são discutidas as implicações dos resultados encontrados.
\end{abstract}

Palavras-chave: Internet; acesso; atitude estudantes universitarios.

Como citar:

Morales, A., Zacatenco, J., Luna, M., García, R., \& Hidalgo, C. (2020). Acceso y actitud del uso de Internet entre jóvenes de educación universitaria. Revista Digital de Investigación en Docencia Universitaria, 14(1), e1174. https://doi. org/10.19083/ridu.2020.1174

F n la última década, el uso de Internet ha ido en aumento (Blázquez et al., 2018) ocupando un importante espacio en la vida cotidiana de las personas, debido a que, actualmente, es utilizado en múltiples contextos con diferentes finalidades (Rodríguez \& Sandoval, 2017). De hecho, son los jóvenes los usuarios más activos (García, 2011; Guan \& Subrahmanyam, 2009; Molero, et al., 2014; Rial, Gómez, Braña, \& Varela, 2014). Diversos estudios, indican que las personas que más utilizan este recurso se encuentran entre los 12 y 34 años de edad (Arredondo, 2007; Cardenas, Paret, Campos \& Campos, 2017; Crovi \& López, 2010; Matellanes, 2011; Molero, et al., 2014; Puerta-Cortés, 2013). Navegan a diario por la red, obtienen información, se comunican por correo, hacen amigos en las redes sociales y en muchas ocasiones son los productores del contenido (Gómez, Roses \& Farias, 2012), y los que van descubriendo el manejo de las aplicaciones que, día con día, nacen en la web.

En el caso de México, el Estudio Nacional sobre la Disponibilidad y Uso de las Tecnologías de Información en los Hogares (ENDUTIH), realizado por el Instituto Nacional de Estadística y Geografía (INEGI) en el 2018, muestra que el 65.8\% de los mexicanos (74.3 millones) son usuarios de Internet, lo cual significó un crecimiento de 3 millones de usuarios con respecto al año anterior. Con edades que van desde seis años en adelante, sin existir una diferencia significativa en cuanto al género. Con relación a los dispositivos más utilizados para acceder a este recurso, se observa en este estudio, que 69.6 millones de personas utilizan Smartphone y 50.8 millones computadora. Lo que, representa un aumento anual del $7.6 \%$ de usuarios de Internet que se conectan a través de 
Smartphone en relación con el año 2017.

Adicionalmente, este estudio identificó que los principales usos que se le dan al Internet son: 1) entretenimiento (90.5\%); 2) comunicación (90.3\%); 3) búsqueda de información (86.9\%); 4) acceso a contenidos audiovisuales (78.1\%); y 5) y redes sociales (77.8\%). También se muestra una menor utilización en las actividades que podrían apoyar en el aspecto educativo, como son: la descarga de software, y la lectura de periódicos, revistas o libros, las cuales representan solo el $49.2 \%$ y $48.7 \%$ respectivamente.

Por otra parte, en el estudio Hábitos de los Usuarios en Internet en México, elaborado por la Asociación de Internet en el 2019, se menciona que más de la mitad de los mexicanos internautas son jóvenes, ya que el $66 \%$ se encuentra entre los 6 y 34 años de edad. Quedando distribuidos de la siguiente manera: a) 12\% tiene entre los 6 y 11 años; b) $14 \%$ se encuentra entre 12 y 17 años; c) $18 \%$ está entre los 18 y 24 años; d) 22\% se sitúa entre los 25 y 34 años; y e) el 34\% se localiza entre los 35 o más años. Además se resalta que la mayor parte de estos usuarios habitan en el centro sur del país (25\%) que comprende la CDMX, Estado de México y Morelos. En contraparte, con los que se ubica en la región sureste formada por los estados de Campeche, Quintana Roo, Tabasco y Yucatán con tan solo un 6\% del total de usuarios de Internet.

En este mismo estudio, se hace un análisis más detallado de los dispositivos utilizados para acceder a Internet. De forma similar a los resultados obtenidos en ENDUTIH, se observa que los usuarios usan con mayor frecuencia el Smartphone (92\%), mientras que el acceso a través de computadoras portátiles (76\%) y de escritorio (48\%) ha disminuido. A la vez que, el uso de las tablets (42\%), aparatos electrónicos (38\%) y los dispositivos wearables (29\%) para conectarse a Internet va en aumento.

Otro aspecto que se destaca es el tiempo de conexión por usuario, ya que se amplió 8 minutos más que en el 2018, arrojando un uso promedio de 8 horas con 20 minutos por día.

En lo que respecta, a los lugares donde se accede a Internet, en el estudio de Hábitos de los Usuarios en Internet en México del 2018, se muestra que se acentúa el hogar (86\%), seguido de cualquier lugar a través del uso de los dispositivos móviles (68\%) y por último en el trabajo (49\%). Aunado a que los métodos de conexión que más se utilizan son: WIFI contratado (56\%), plan de datos (39\%) y prepago (30\%). Además, este estudio reporta que en México las principales razones de acceso a Internet son el uso de redes sociales (82\%), la mensajería instantánea (78\%) y el envío-recepción de correos electrónicos (77\%). En base a estos resultados, se puede decir que los mexicanos utilizan el Internet principalmente como medio de socialización y comunicación. En segundo lugar, se encuentra la búsqueda de información (76\%). Y en tercer lugar, se enfatiza la utilización de mapas (68\%), ver películas (65\%) y leer contenido relevante (59\%).

Con lo anterior, se observa que el uso del Internet es casi ilimitado y se está generalizando en la población mexicana, más allá de las barreras demográficas y socioeconómicas, al igual que el número de usuarios que hacen uso de las redes sociales, del correo electrónico, de la mensajería instantánea, de los cursos en línea, etcétera.

Sin embargo, estos estudios, tanto el que realizó el INEGI en el año 2018, como la Asociación de Internet en los años 2018 y 2019, aún no muestran cuáles son las prácticas habituales del uso de Internet en la vida cotidiana de los principales actores (estudiantes) del sistema educativo en México. Tampoco se precisa si este uso afecta de alguna manera su desempeño académico, a pesar de que, desde hace varios años, el Internet se está introduciendo en el mundo de la educación como un instrumento potente para el trabajo académico, y la formación profesional y técnica. Lo anterior, se debe a que "el Internet favorece el autoaprendizaje, el trabajo en equipo, la comunicación interpersonal, la retroalimentación, el contacto con expertos, así como el acceso a diferentes fuentes de información y contenidos que apoyan e incluso facilitan el aprendizaje constructivista y colaborativo" (Gómez et al., 2012, p.65), etc.

Esto se suma a que el cúmulo de investigaciones científicas realizadas en México, que focalizan su atención en torno a esta temática, son todavía limitadas y, en su mayoría, refieren a casos concretos y poblaciones específicas (Arredondo, 2007; Crovi \& López, 2010; Cortázar, 2004; Ortiz \& Gallego, 2009; Ramírez, Maldonado \& Marín, 2015; Sánchez, et al., 2006). Por ello, el objetivo de la presente investigación es conocer si los estudiantes de nivel universitario utilizan el Internet, y cuáles son sus prácticas habituales de acceso; además de 
identificar el nivel de actitud hacia los diversos usos que le aplican y si este se asocia con el género, especialidad, semestre y rendimiento académico.

Lo anterior permitirá contar con información relevante para que, en un futuro, se puedan programar estrategias de implementación para mejorar el uso de Internet en el área académica o prevenir algún factor de riesgo que sea ocasionado por un uso excesivo.

\section{Método}

\section{Diseño}

Se realizó un estudio de tipo cuantitativo con un enfoque descriptivo, debido a que se explicitaron las características, propiedades y rasgos más importantes del fenómeno investigado; transversal, ya que se obtuvieron resultados de una población específica en un tiempo determinado; por último, correlacional, porque se evalúo la relación que existe entre las variables.

\section{Participantes}

La muestra fue no probabilística y por conveniencia con un total de 813 participantes (520 mujeres y 293 hombres) con un rango de edad que oscilaba entre los 18 y 24 años $\left(M_{\text {edad }}=20.45\right.$ años, $D E_{\text {edad }}=$ 1.70) de una universidad pública del Estado de México, divididos en seis especialidades: 170 fueron estudiantes de Psicología (LPS), 153 mujeres y 17 hombres; 151 de Derecho (LDE), 108 mujeres y 43 hombres; 150 de Ingeniería en Computación (ICO), 51 mujeres y 99 hombres; 119 de Informática Administrativa (LIA), 65 mujeres y 54 varones; 112 de Contaduría (LCN), 74 mujeres y 38 hombres; y 111 de Administración (LAM), 69 mujeres y 42 hombres.

\section{Instrumento}

Se utilizó una encuesta compuesta por dos secciones. En la primera sección, se les solicitó a los participantes información demográfica (edad, género, semestre, especialidad y promedio general) y frecuencias de acceso a Internet. En la segunda sección, se aplicó la escala 5-T (Chien, Sen-Chi, Chao-Hsiu \& Huan-Chuehu, 2009), que consta de 42 ítems redactados mediante afirmaciones presentadas en una escala tipo Likert con cuatro opiniones de respuesta ( $1=$ totalmente en desacuerdo; hasta $4=$ totalmente de acuerdo), en donde puntaciones altas indican una actitud más positiva hacia los diferentes usos de Internet con una consistencia interna total excelente de $\mathrm{a}=\mathbf{9 5}$. La escala $5 \mathrm{~T}$ consta de cinco factores que se describen a continuación:

- El factor Fuente de Información está compuesto por 9 ítems, los cuales hacen referencia a la recopilación de información tanto general para la rutina diaria, como especializada y científica para expandir conocimientos y realizar trabajos escolares.

- El factor Territorio incluye 8 ítems, que corresponden al empleo de Internet para que, a través de diversos grupos (redes sociales y blogs), los usuarios puedan expresar sentimientos, publicar contenido e intercambiar ideas y opiniones sobre intereses similares o trabajos escolares.

- El factor Teléfono está constituido por 6 ítems, que conciernen al uso de Internet como medio de comunicación entre compañeros, amigos, profesores y familiares.

- El factor Juguete está conformado por 14 ítems, los cuales hacen referencia al uso de Internet para el entretenimiento, ocio y el juego en línea.

- El factor Herramienta contiene 5 ítems, que corresponden al empleo de Internet como apoyo para realizar tareas escolares y como ayuda para aprender a utilizar nuevas aplicaciones durante el ciclo escolar.

Para la presente investigación, se consideró al rendimiento académico como el resultado que obtuvieron los estudiantes en el cumplimiento de los objetivos establecidos en los programas educativos y que es expresado por el promedio que han acumulado durante los semestres cursados. Cabe señalar, que este fue otorgado por cada participante durante la aplicación de la encuesta, debido a que la política de protección de datos personales de la propia universidad, no permite al personal del departamento de control escolar proporcionar datos de los estudiantes a terceros.

\section{Procedimiento}

Se invitó a los estudiantes a participar en la encuesta con una previa autorización de las autori- 
dades de la universidad. La encuesta fue administrada a través de una plataforma en línea y se les explicaron los objetivos generales del estudio, así como las instrucciones para acceder y responder a los ítems. Posteriormente, se les mostró el consentimiento informado. Luego de leerlo y aceptarlo, se habilitaron las dos secciones del instrumento para ser contestadas. La participación fue voluntaria y el tiempo para la resolución de los ítems osciló entre los 15 y 20 minutos.

\section{Análisis de datos}

Los datos fueron procesados con el Paquete Estadístico para las Ciencias Sociales (SPSS, por sus siglas en inglés) versión 20, aplicando los siguientes análisis: a) análisis descriptivos (medias y desviación estándar), b) análisis de fiabilidad (coeficiente alfa de Cronbach), c) análisis con medias (prueba $t$ de Student, ANOVA con comparaciones post hoc Tukey, y medida de efecto $d$ de Cohen y eta cuadrado) y d) análisis de correlaciones (coeficiente de correlación de Pearson).

\section{Resultados}

\section{Hábitos de uso y acceso a Internet}

En lo que se refiere al uso y acceso, se observa que el 74.7\% de los jóvenes universitarios reconoce llevar 5 años o más utilizando Internet, es decir, 7 de cada 10 navegan en la red desde hace ya varios años. A estos habría que añadir un 23.2\% que lleva conectándose desde hace 3 y 4 años. Se conectan, por lo general, diariamente durante 4 horas o más (73.6\%) y lo hacen masivamente desde su propio hogar (89.5\%); aunque un 5.7\% lo hacen en la escuela; el $2.2 \%$ en lugares públicos; y el $2.6 \%$, en los hogares de otras personas (tabla 1).

\section{Estadísticos descriptivos}

Los hallazgos de la confiabilidad incluidos en la tabla 2 indican que se alcanzaron coeficientes alfa aceptables, los cuales fluctuaron entre .73 y .89; y un nivel de consistencia interna total adecuada $\mathrm{a}=.93$ (Nunnaly, 1978). Además, se puede apreciar que los cinco factores correlacionaron de forma estadísticamente significativa. Este resultado sugiere que no son excluyentes.

Por otro lado, los resultados generales de las medias y desviaciones estándar muestran que los factores Fuente de Información ( $M=3.01 ; \mathrm{DE}=0.50)$ y Herramienta $(M=3.16 ; \mathrm{DE}=0.53)$ se encuentran en un nivel de actitud más intenso en comparación con los factores Territorio $(M=2.60 ; \mathrm{DE}=0.52)$, Teléfono $(M=2.39$; DE=0.59) y Juguete $(M=2.56$; $\mathrm{DE}=0.56$ ) que se ubican en un nivel de actitud menor, indicativo de que los universitarios muestran una tendencia de actitud de uso de Internet mucho mayor tanto para la búsqueda de información general o científica como para realizar trabajos y tareas durante el ciclo escolar, por sobre el uso de este para la comunicación, el entretenimiento, las redes sociales, el ocio y los juegos en línea (tabla 2).

\section{Tabla 1}

Frecuencias de uso y acceso a Internet

\begin{tabular}{|l|l|c|}
\hline \multicolumn{2}{|c|}{ Frecuencias de acceso a Internet } & Porcentajes \\
\hline \multirow{4}{*}{ Años de uso } & Menos de un año & 0.5 \\
\cline { 2 - 3 } & 1 a 2 años & 1.6 \\
\cline { 2 - 3 } & 3 a 4 años & 23.2 \\
\cline { 2 - 3 } & 5 años o mas & 74.7 \\
\hline \multirow{2}{*}{ Tiempo de conexión diario } & 1 hr. & 4.1 \\
\cline { 2 - 3 } & 2 a 3 hrs. & 22.4 \\
\cline { 2 - 3 } & 4 a 5 hrs. & 38.5 \\
\cline { 2 - 3 } & 6 hrs. o más & 35.1 \\
\hline \multirow{2}{*}{ Lugar de conexión } & Hogar & 89.5 \\
\cline { 2 - 3 } & Escuela & 5.7 \\
\cline { 2 - 3 } & Lugares públicos & 2.2 \\
\cline { 2 - 3 } & Hogares de otras personas & 2.6 \\
\hline
\end{tabular}


Tabla 2

Estadísticos descriptivos, correlaciones y fiabilidad

\begin{tabular}{|c|c|c|c|c|c|c|c|c|}
\hline Factor & $\boldsymbol{M}$ & $D E$ & $\begin{array}{c}\text { Fuente de } \\
\text { Información }\end{array}$ & Territorio & Teléfono & Juguete & Herramienta & Escala Total \\
\hline $\begin{array}{l}\text { Fuente de } \\
\text { Información }\end{array}$ & 3.01 & 0.50 & $(0.84)$ & & & & & \\
\hline Territorio & 2.60 & 0.52 & $0.50^{*}$ & $(0.82)$ & & & & \\
\hline Teléfono & 2.39 & 0.59 & $0.30^{* *}$ & $0.52 *$ & (0.73) & & & \\
\hline Juguete & 2.56 & 0.56 & $0.46^{*}$ & $0.55^{*}$ & $0.63^{*}$ & (0.89) & & \\
\hline Herramienta & 3.16 & 0.53 & $0.63^{*}$ & $0.39 *$ & $0.32 *$ & $0.49^{*}$ & $(0.76)$ & \\
\hline Escala Total & 2.70 & 0.42 & $0.73^{* *}$ & $0.76^{*}$ & $0.72 *$ & 0.89" & $0.68^{*}$ & $(0.93)$ \\
\hline
\end{tabular}

** La correlación es significativa al nivel 0.01 (bilateral).

Los coeficientes de alfa de Cronbach se presentan entre paréntesis

\section{Usos de Internet por género}

Atendiendo al género, se observa la existencia de diferencias significativas entre los grupos (tabla 3) con un tamaño del efecto ( $d$ Cohen) de intensidad media. Concretamente, a la hora de utilizar la Internet como juguete, son los hombres ( $M=2.72$, $D E=0.55, \mathrm{p}<0.05, d=0.47)$ los que presentan una actitud más positiva para jugar en línea y como instrumento de ocio en contraste con las mujeres ( $M=2.47, D E=0.55$ ). No obstante, tanto los hombres como las mujeres exteriorizan tener casi el mismo nivel de uso en los otros factores (tabla 3).

\section{Usos de Internet por especialidad}

Teniendo en cuenta las puntuaciones medias en cada uno de los factores en función de la variable especialidad (tabla 4), se dan diferencias significativas según el análisis de varianza y la prueba post hoc Tukey, con un tamaño del efecto pequeño (Eta cuadrado). Los universitarios de ICO son los que muestra tener una actitud más positiva hacia los factores Fuente de Información $(M=3.09, D E=0.46$, $\left.\mathrm{p}<0.05, \mathrm{~h}^{2}=0.01\right)$, Territorio $(=2.67, D E=0.44, \mathrm{p}<0.01$, $\left.\mathrm{h}^{2}=0.02\right)$, Juguete $\left(=2.79, D E=0.54, \mathrm{p}<0.01, \mathrm{~h}^{2}=0.04\right)$ y Herramienta $\left(M=3.31, D E=0.44, \mathrm{p}<0.0, \mathrm{~h}^{2}=0.03\right.$ ) por encima de la actitud que presentan los estudiantes que pertenecen a otras carreras. Lo anterior podría deberse al perfil académico de los universitarios, ya que los ingenieros en computación deben ser especialistas en el funcionamiento y desarrollo de las tecnologías de información y comunicación incluyendo la Internet.

Sin embargo, no se encontraron diferencias entre las especialidades en el factor teléfono. En otras palabras, todos los estudiantes, independientemente de la disciplina a la que pertenezcan, reconocen por igual tener el mismo nivel de actitud para utilizar la Internet como un medio de comunicación con sus amigos, familiares y/o profesores (tabla 4).

Tabla 3

Usos de Internet por género

\begin{tabular}{|c|c|c|c|c|c|c|}
\hline \multirow{2}{*}{ Factores } & \multicolumn{2}{|c|}{ Mujer } & \multicolumn{2}{|c|}{ Hombre } & \multirow[b]{2}{*}{$t$} & \multirow[b]{2}{*}{$\boldsymbol{p}$} \\
\hline & $M$ & $D E$ & $\boldsymbol{M}$ & $D E$ & & \\
\hline Fuente de Información & 2.99 & 0.50 & 3.04 & 0.50 & 0.30 & 0.17 \\
\hline Territorio & 2.58 & 0.54 & 2.64 & 0.50 & 2.04 & 0.08 \\
\hline Teléfono & 2.37 & 0.58 & 2.43 & 0.60 & 0.28 & 0.15 \\
\hline Juguete & 2.47 & 0.55 & 2.72 & 0.55 & 0.20 & $0.00^{\circ}$ \\
\hline Herramienta & 3.15 & 0.54 & 3.19 & 0.51 & 0.51 & 0.20 \\
\hline
\end{tabular}

$M=$ media; $D E=$ desviación estándar; $t=$ prueba $t$ de student $;{ }^{*} p<0.5$. 
Tabla 4

Usos de Internet por licenciatura

\begin{tabular}{|c|c|c|c|c|c|c|c|c|}
\hline \multirow{2}{*}{ Factores } & ICO & LIA & LAM & LPS & LCN & LDE & \multirow{2}{*}{$\begin{array}{c}F \\
(5,807)\end{array}$} & \multirow{2}{*}{$\boldsymbol{p}$} \\
\hline & $\begin{array}{c}M \\
D E\end{array}$ & $\begin{array}{l}M \\
D E\end{array}$ & $\begin{array}{l}M \\
D E\end{array}$ & $\begin{array}{c}M \\
D E\end{array}$ & $\begin{array}{l}M \\
D E\end{array}$ & $\begin{array}{l}M \\
D E\end{array}$ & & \\
\hline Fuente de Información & $\begin{array}{c}3.09 \\
(0.46)\end{array}$ & $\begin{array}{c}3.00 \\
(0.45)\end{array}$ & $\begin{array}{c}3.01 \\
(0.47)\end{array}$ & $\begin{array}{c}2.91 \\
(0.57)\end{array}$ & $\begin{array}{l}3.00 \\
(0.51)\end{array}$ & $\begin{array}{c}3.05 \\
(0.50)\end{array}$ & 2.30 & $0.04^{\circ}$ \\
\hline Territorio & $\begin{array}{c}2.67 \\
(0.49)\end{array}$ & $\begin{array}{c}2.62 \\
(0.53)\end{array}$ & $\begin{array}{c}2.58 \\
(0.49)\end{array}$ & $\begin{array}{c}2.47 \\
(0.52)\end{array}$ & $\begin{array}{c}2.58 \\
(0.55)\end{array}$ & $\begin{array}{c}2.62 \\
(0.53)\end{array}$ & 3.41 & $0.00^{\circ}$ \\
\hline Teléfono & $\begin{array}{c}2.44 \\
(0.59)\end{array}$ & $\begin{array}{c}2.35 \\
(0.58)\end{array}$ & $\begin{array}{c}2.37 \\
(0.54)\end{array}$ & $\begin{array}{c}2.34 \\
(0.55)\end{array}$ & $\begin{array}{c}2.44 \\
(0.60)\end{array}$ & $\begin{array}{c}2.42 \\
(0.63)\end{array}$ & 0.86 & 0.51 \\
\hline Juguete & $\begin{array}{c}2.79 \\
(0.54)\end{array}$ & $\begin{array}{c}2.52 \\
(0.55)\end{array}$ & $\begin{array}{c}2.53 \\
(0.55)\end{array}$ & $\begin{array}{l}2.44 \\
(0.51)\end{array}$ & $\begin{array}{c}2.54 \\
(0.55)\end{array}$ & $\begin{array}{c}2.54 \\
(0.59)\end{array}$ & 7.30 & $0.00^{\circ}$ \\
\hline Herramienta & $\begin{array}{c}3.31 \\
(0.44)\end{array}$ & $\begin{array}{c}3.20 \\
(0.55)\end{array}$ & $\begin{array}{c}3.07 \\
(0.46)\end{array}$ & $\begin{array}{c}3.05 \\
(0.63)\end{array}$ & $\begin{array}{c}3.21 \\
(0.48)\end{array}$ & $\begin{array}{c}3.14 \\
(0.50)\end{array}$ & 4.94 & $0.00^{\circ}$ \\
\hline
\end{tabular}

$M=$ media; $D E=$ desviación estándar; $F=$ prueba de Fisher; ${ }^{*} p<.05$.

\section{Usos de Internet por rendimiento académico}

Al comparar los grupos según su promedio general obtenido hasta el momento en la especialidad (tabla 5), se muestran diferencias significativas. Los estudiantes que declaran un promedio más bajo (entre 7.0 y 7.9) presentan actitudes más positivas hacia los factores Territorio $(M=2.64$, $\left.D E=0.51, \mathrm{p}<0.05, \mathrm{~h}^{2}=0.01\right)$ y Juguete $(M=2.64, D E$ $\left.=0.53, \mathrm{p}<0.05, \mathrm{~h}^{2}=0.01\right)$ en comparación con los estudiantes que cuentan con promedios más altos. En otras palabras, los alumnos con promedio más bajo prefieren jugar más en línea y publicar contenido en sus páginas web, blogs y redes sociales, que los alumnos con promedios más altos. No obstante, a pesar de la presencia de diferen- cias significativas entre los grupos, el tamaño del efecto fue de intensidad pequeña, según los valores de Eta cuadrado (tabla 5).

\section{Usos de Internet por edad}

Relacionando la edad de los estudiantes con la media obtenida en cada uno de los factores, se puede apreciar que su actitud hacia el uso de Internet como Fuente de información va en aumento conforme a la edad. Además se muestra una diferencia significativa (tabla 6), con un tamaño del efecto de intensidad pequeña (Eta cuadrado). Los estudiantes de 21 años ( $M=2.66, \mathrm{DE}=0.56, \mathrm{p}<0.05, \mathrm{~h} 2=0.01)$ tienden a utilizar un poco más el Internet como Juguete en comparación con compañeros de otras edades (tabla 6).

Tabla 5

Comparación por promedio

\begin{tabular}{|c|c|c|c|c|c|}
\hline \multirow{2}{*}{ Factores } & 7.0-7.9 & 8.0-8.9 & 9.0-10 & \multirow{2}{*}{$\begin{array}{c}F \\
(2,810)\end{array}$} & \multirow[b]{2}{*}{$\boldsymbol{p}$} \\
\hline & $\begin{array}{c}M \\
D E\end{array}$ & $\begin{array}{c}M \\
D E\end{array}$ & $\begin{array}{c}M \\
D E\end{array}$ & & \\
\hline Fuente de Información & $\begin{array}{c}2.98 \\
(0.47)\end{array}$ & $\begin{array}{c}3.02 \\
(0.49)\end{array}$ & $\begin{array}{c}2.99 \\
(0.54)\end{array}$ & 0.60 & 0.54 \\
\hline Territorio & $\begin{array}{c}2.64 \\
(0.51)\end{array}$ & $\begin{array}{c}2.62 \\
(0.52)\end{array}$ & $\begin{array}{c}2.48 \\
(0.52)\end{array}$ & 5.75 & $0.00^{\circ}$ \\
\hline Teléfono & $\begin{array}{c}2.42 \\
(0.56)\end{array}$ & $\begin{array}{c}2.41 \\
(0.58)\end{array}$ & $\begin{array}{c}2.31 \\
(0.59)\end{array}$ & 2.29 & 0.10 \\
\hline Juguete & $\begin{array}{c}2.64 \\
(0.53)\end{array}$ & $\begin{array}{c}2.57 \\
(0.55)\end{array}$ & $\begin{array}{c}2.47 \\
(0.59)\end{array}$ & 4.54 & $0.01^{\circ}$ \\
\hline Herramienta & $\begin{array}{c}3.15 \\
(0.51)\end{array}$ & $\begin{array}{c}3.17 \\
(0.51)\end{array}$ & $\begin{array}{c}3.13 \\
(0.58)\end{array}$ & 0.44 & 0.64 \\
\hline
\end{tabular}

$M=$ media; $D E=$ desviación estándar; $F=$ prueba de Fisher; ${ }^{*} p<.05$. 
Tabla 6

Comparación por edad

\begin{tabular}{|c|c|c|c|c|c|c|c|c|}
\hline \multirow{2}{*}{ Factores } & 18 años & 19 años & 20 años & 21 años & 22 años & $\begin{array}{l}23 \text { años } \\
\text { o más }\end{array}$ & \multirow{2}{*}{$\begin{array}{c}\boldsymbol{F} \\
(5,807)\end{array}$} & \multirow{2}{*}{$p$} \\
\hline & $\begin{array}{l}M \\
D E\end{array}$ & $\begin{array}{l}M \\
D E\end{array}$ & $\begin{array}{l}M \\
D E\end{array}$ & $\begin{array}{l}M \\
D E\end{array}$ & $\begin{array}{l}M \\
D E\end{array}$ & $\begin{array}{l}M \\
D E\end{array}$ & & \\
\hline Fuente de Información & $\begin{array}{c}2.95 \\
(0.54)\end{array}$ & $\begin{array}{c}3.03 \\
(0.46)\end{array}$ & $\begin{array}{c}2.95 \\
(0.52)\end{array}$ & $\begin{array}{c}3.01 \\
(0.50)\end{array}$ & $\begin{array}{c}3.03 \\
(0.51)\end{array}$ & $\begin{array}{c}3.08 \\
(0.47)\end{array}$ & 1.35 & 0.24 \\
\hline Territorio & $\begin{array}{c}2.64 \\
(0.50)\end{array}$ & $\begin{array}{c}2.57 \\
(0.50)\end{array}$ & $\begin{array}{c}2.55 \\
(0 . .53)\end{array}$ & $\begin{array}{c}2.68 \\
(0.54)\end{array}$ & $\begin{array}{c}2.51 \\
(0.47)\end{array}$ & $\begin{array}{c}2.58 \\
(0.54)\end{array}$ & 1.90 & 0.09 \\
\hline Teléfono & $\begin{array}{c}2.48 \\
(0.54)\end{array}$ & $\begin{array}{c}2.42 \\
(0.56)\end{array}$ & $\begin{array}{c}2.34 \\
(0.56)\end{array}$ & $\begin{array}{c}2.43 \\
(0.62)\end{array}$ & $\begin{array}{c}2.25 \\
(0.56)\end{array}$ & $\begin{array}{c}2.37 \\
(0.61)\end{array}$ & 1.92 & 0.09 \\
\hline Juguete & $\begin{array}{c}2.57 \\
(0.58)\end{array}$ & $\begin{array}{c}2.53 \\
(0.54)\end{array}$ & $\begin{array}{c}2.55 \\
(0.54)\end{array}$ & $\begin{array}{c}2.66 \\
(0.56)\end{array}$ & $\begin{array}{c}2.42 \\
(0.59)\end{array}$ & $\begin{array}{c}2.54 \\
(0.54)\end{array}$ & 2.30 & $0.04^{*}$ \\
\hline Herramienta & $\begin{array}{c}3.10 \\
(0.52)\end{array}$ & $\begin{array}{c}3.10 \\
(0.51)\end{array}$ & $\begin{array}{c}3.16 \\
(0.58)\end{array}$ & $\begin{array}{c}3.22 \\
(0.52)\end{array}$ & $\begin{array}{c}3.12 \\
(0.55)\end{array}$ & $\begin{array}{c}3.24 \\
(0.45)\end{array}$ & 1.84 & 0.10 \\
\hline
\end{tabular}

$M=$ media; $D E=$ desviación estándar; $F=$ prueba de Fisher; ${ }^{*} p<.05$.

\section{Uso de Internet por semestre}

Teniendo en cuenta las puntuaciones medias en cada uno de los factores con respecto a la variable semestre, se puede observar en la tabla 7, que hay diferencias en los factores Teléfono y Juguete, este último con un tamaño del efecto de intensidad pequeña (Eta cuadrado). Los estudiantes de segundo semestre son los que manifiestan puntuaciones más elevadas a la hora de utilizar el Internet como un medio de comunicación con otras personas $(M=2.44, D E=0.57)$ y como herramienta de entretenimiento $(M=2.62, D E=0.57$, $\mathrm{p}<0.05, \mathrm{~h}^{2}=0.01$ ) en comparación con los de décimo semestre (tabla 7).

\section{Correlación entre factores,}

\section{edad y rendimiento académico}

Se realizó una correlación de Pearson para conocer si existe una relación entre los diversos factores del instrumento, la edad y el promedio de los universitarios (tabla 8). Se observó que, a pesar de que algunos niveles de significancia se encuentran entre 0.01 y 0.05 bilateral, no se han obtenido valores razonables para establecer una correlación lineal. De hecho, las correlaciones de Pearson son muy bajas: edad con el factor Herramienta 0.08; y el rendimiento académico con el factor Territorio -0.10 y Juguete -0.10. Por tanto, no existe correlación entre las variables (tabla 8 ).

Tabla 7

Comparación por semestre

\begin{tabular}{|c|c|c|c|c|c|c|c|}
\hline \multirow[b]{2}{*}{ Factores } & $2^{\circ}$ & $4^{\circ}$ & $6^{\circ}$ & $8^{\circ}$ & $10^{\circ}$ & \multirow{2}{*}{$\begin{array}{c}F \\
(4,808)\end{array}$} & \multirow[b]{2}{*}{$\boldsymbol{P}$} \\
\hline & $\begin{array}{c}M \\
D E\end{array}$ & $\begin{array}{c}M \\
D E\end{array}$ & $\begin{array}{l}M \\
D E\end{array}$ & $\begin{array}{l}M \\
D E\end{array}$ & $\begin{array}{c}M \\
D E\end{array}$ & & \\
\hline Tesoro de la información & $\begin{array}{c}3.00 \\
(0.51)\end{array}$ & $\begin{array}{c}2.98 \\
(0.48)\end{array}$ & $\begin{array}{c}3.01 \\
(0.46)\end{array}$ & $\begin{array}{c}3.06 \\
(0.54)\end{array}$ & $\begin{array}{c}3.01 \\
(0.53)\end{array}$ & 0.53 & 0.70 \\
\hline Territorio & $\begin{array}{c}2.62 \\
(0.51)\end{array}$ & $\begin{array}{c}2.58 \\
(0.47)\end{array}$ & $\begin{array}{c}2.56 \\
(0.53)\end{array}$ & $\begin{array}{c}2.63 \\
(0.56)\end{array}$ & $\begin{array}{c}2.60 \\
(0.61)\end{array}$ & 0.50 & 0.73 \\
\hline Teléfono & $\begin{array}{c}2.44 \\
(0.57)\end{array}$ & $\begin{array}{c}2.40 \\
(0.53)\end{array}$ & $\begin{array}{c}2.37 \\
(0.55)\end{array}$ & $\begin{array}{c}2.33 \\
(0.69) \\
\end{array}$ & $\begin{array}{c}2.32 \\
(0.62) \\
\end{array}$ & 0.94 & 0.44 \\
\hline Juguete & $\begin{array}{c}2.62 \\
(0.57)\end{array}$ & $\begin{array}{c}2.53 \\
(0.52)\end{array}$ & $\begin{array}{c}2.61 \\
(0.52)\end{array}$ & $\begin{array}{c}2.50 \\
(0.62)\end{array}$ & $\begin{array}{c}2.40 \\
(0.54)\end{array}$ & 2.50 & $0.04^{\circ}$ \\
\hline Herramienta & $\begin{array}{c}3.13 \\
(0.53)\end{array}$ & $\begin{array}{c}3.12 \\
(0.51)\end{array}$ & $\begin{array}{c}3.23 \\
(0.52)\end{array}$ & $\begin{array}{c}3.20 \\
(0.53)\end{array}$ & $\begin{array}{c}3.16 \\
(0.55)\end{array}$ & 1.56 & 0.18 \\
\hline
\end{tabular}

$M=$ media; $D E=$ desviación estándar $F=$ prueba de Fisher; ${ }^{*} p<.05$. 
Tabla 8

Compración por factores, edad y rendimiento académico

\begin{tabular}{|c|c|c|c|c|c|c|}
\hline \multicolumn{2}{|c|}{} & $\begin{array}{c}\text { Fuente de } \\
\text { Información }\end{array}$ & Territorio & Teléfono & Juguete & Herramienta \\
\hline \multirow{2}{*}{ Edad } & $\begin{array}{c}\text { Correlación de } \\
\text { Pearson }\end{array}$ & 0.06 & -0.02 & -0.07 & -0.02 & $0.08^{*}$ \\
\cline { 2 - 7 } & Sig. & - & - & - & - & 0.03 \\
\hline \multirow{2}{*}{$\begin{array}{c}\text { Rendimiento } \\
\text { académico }\end{array}$} & $\begin{array}{c}\text { Correlación de } \\
\text { Pearson }\end{array}$ & 0.00 & -0.10 & -0.06 & $-0.10^{* *}$ & -0.01 \\
\cline { 2 - 7 } & Sig. & - & 0.00 & - & 0.00 & - \\
\hline
\end{tabular}

** La correlación es significativa al nivel 0.01 (bilateral) y *significativa al nivel .05.

\section{Discusión y Conclusión}

Los resultados obtenidos en este estudio muestran que, desde hace varios años (más de cinco), el uso de Internet es masivo entre los universitarios estudiados sin presentar diferencias por género. Acceden a la red principalmente desde su propio hogar, aunque una pequeña parte lo hace desde la escuela, lugares públicos o en hogares de otras personas, por un tiempo de cuatro horas o más diariamente. Estos datos coinciden con los resultados obtenidos por Puerta-Cortes y Carbonell (2013) y García (2011), pero difieren de los datos reportados años atrás, ya que los estudiantes se conectaban como máximo una vez al día o a la semana fundamentalmente desde un cibercafé seguido de su hogar o desde la universidad (Rodrigues, Canales, Peña, Castro \& Reyes, 2009). Estos datos muestran el veloz incremento del uso de Internet por parte de los estudiantes.

Si bien tanto las mujeres como los hombres exteriorizan un intenso uso de Internet, se muestran diferencias importantes en cuanto al nivel de actitud por el tipo de contenido al que acceden. La actitud de los hombres es más positiva cuando utilizan el Internet para jugar en línea y como instrumento de entretenimiento y ocio (factor Juguete) en contraste con las mujeres. Estos resultados avalan los datos encontrados en este sentido en algunos estudios previos (Fernández-de-Arroyabe-Olaortua, Lazkano-Arrillaga, \& Eguskiza-Sesumaga, 2018; Torres-Díaz, Duart, Gómez-Alvarado, Marín-Gutiérrez, \& Segarra-Faggioni, 2016; Fernández, Peñalba \& Irazabal, 2015; Ruiz-Olivares, Lucena, Pino \& Herruzo, 2010). Por ello, la probabilidad de que ellas presenten una posible adicción lúdica virtual parece ser menor.

De igual manera, se encontraron diferencias entre las edades y los semestres con el factor Juguete. Los universitarios de 21 años y los que se encuentran inscritos en segundo semestre son los que mayor puntuación obtuvieron en comparación con los que tienen 22 años y están estudiando el último semestre de la carrera. Es decir, tener 21 años y estar cursando el segundo semestre de la carrera, pueden ser características que determine un nivel de actitud más positiva en el uso de Internet para jugar en línea y como un instrumento para matar el tiempo. En virtud de estas cifras, se puede concluir que algunos estudiantes pueden no dar tanta prioridad al éxito académico durante sus primeros años de escuela.

Por otra parte, los resultados también muestran que no existe una relación entre el rendimiento académico de los universitarios, la edad y los cinco factores. Por ende, a medida que aumenta la edad y el promedio, el nivel de intensidad del uso de Internet puede aumentar o disminuir. Estos datos se contrastan con la investigación de Torres-Díaz, et al. (2016), en donde se menciona que existe una incidencia positiva del uso de Internet para el entretenimiento sobre el rendimiento académico. Mientras que Türel \& Toraman (2015) afirman en su investigación que el uso excesivo de Internet, incluso si no está en un nivel adictivo, se correlaciona negativamente con el éxito académico de los estudiantes.

Conocer los niveles de acceso y la actitud de los diferentes usos que los universitarios hacen del Internet se vuelve un trabajo inevitable, debido a que nos encontramos en una sociedad caracte- 
rizada por la constante evolución tecnológica. El contar con información oportuna a nivel preventivo puede facilitar el diseño de planes de acción para crear conciencia sobre los riesgos derivados de un nivel de uso excesivo, o por otro lado, se podría aprovechar este uso para que el Internet pueda ser promovido de manera correcta en el área académica de cada licenciatura aprovechando las ventajas que este ofrece en el entorno escolar.

El Internet, al ser parte de las TIC, no debe ser incorporado dentro de los procesos de enseñanza-aprendizaje como un elemento aislado e independiente, sino como un fragmento de un crisol de elementos (objetivos, metodologías, aspectos organizativos, contenidos, características de los estudiantes, etcétera) que permitan trazar estrategias diferentes o más exitosas, y no solo usarlo para buscar información y hacer lo mismo que se realizaba sin él (Cabero, 2015).

Se considera que los hallazgos encontrados en el presente estudio tienen implicaciones importantes para estudiantes, padres de familia y autoridades académicas. Sin embargo, en el futuro se sugiere no limitarse en solo indagar como se utiliza el Internet por parte de los estudiantes y si tiene alguna relación con su rendimiento académico, sino su impacto cuando este es aplicado como un recurso didáctico en el proceso de enseñanza-aprendizaje dentro y fuera de las instituciones educativas.

\section{Referencias}

Arredondo, P. (2007). Acceso y usos de internet en el occidente de México: el caso de Jalisco. Comunicación y Sociedad, (8), 11-33. Recuperado de http://bit. ly/2AzvAwH

Asociación de Internet. (2018). $14^{\circ}$ Estudio sobre los hábitos de los usuarios de Internet en México. Recuperado de http://bit.ly/2DNqeBs

Asociación de Internet. (2019). $15^{\circ}$ Estudio sobre los hábitos de los usuarios de Internet en México. Recuperado de http://bit.ly/2YSZaps

Blázquez, B. M., Gómez, R. D., Frontaura F. I., Camacho O. A., Rodríguez, S. F., \& Toriz, C. H. (2018). Uso de Internet por los adolescentes en la búsqueda de información sanitaria. Atención Primaria, 50(9), 547-552. https:// doi.org/10.1016/j.aprim.2017.06.005

Cabero (2015). Reflexiones educativas sobre las tecnologías de la información y comunicación (TIC). Tecnología, Ciencia y Educación, 1, 19-27. Recuperado de http://bit.ly/2BGV39t

Cardenas, B., Paret, M., Campos, P. R., \& Campos, P. G. (2017) Mediciones en los usos sociales de Internet por estudiantes universitarios cubanos de Ciencias de la Información, Periodismo y Comunicación Social. Revista Cubana de Información en Ciencias de la Salud. 28(2), 1-20. Recuperado de http://bit.ly/36yXCa5

Chien, Sen-chi, Chao-Hsiu, \& Huan-Chuehu (2009). Tool, Toy, Telephone, Territory or Treasure of Information: Elemetary school student's attitudes toward the Internet. Computer \& Education 53(2), 308-316. https://doi.org/10.1016/j.compedu.2009.02.003

Cortázar, F. (2004). Chicanos y mexicoamericanos en tres comunidades electrónicas. Comunicación y Sociedad, (2), 125-159. Recuperado de http://bit.ly/2BGV39t

Crovi, D., \& López, R. (2010). Tejiendo voces: Jóvenes universitarios opinan sobre la apropiación de internet en la vida académica. Revista Mexicana de Ciencias Políticas y Sociales, 56(212), 69-80. Recuperado de http://bit.ly/2zxTQzU

Fernández-de-Arroyabe-Olaortua, A., Lazkano-Arrillaga, I., \& Eguskiza-Sesumaga, L. (2018). Nativos digitales: Consumo, creación y difusión de contenidos audiovisuales. Comunicar, 26(57), 61-69. https://doi. org/10.3916/C57-2018-06

Fernández, M., Peñalba, A., \& Irazabal, I. (2015). Hábitos de uso y conductas de riesgo en Internet en la preadolescencia. Comunicar, 22(44), 113-120. https://doi. org/10.3916/C44-2015-12

García, A. (2011). Una perspectiva sobre los riesgos y usos de Internet en la adolescencia. ICONO14 Revista Científica de Comunicación y Tecnologías Emergentes, 9(3), 410-425. https://doi.org/10.7195/ri14. v9i3.62

Gómez, M., Roses, S., \& Farias, P. (2012). El uso académico de las redes sociales en universitarios. Comunicar, 19(38), 131-138. doi: https://doi/org/10.3916/C38 2011-03-04.

Guan, S., \& Subrahmanyam, K. (2009). Youth Internet use: risks and opportunities. Current Opinion in Psychiatry, 22(4), 351-356. https://doi/org/10.1097/YCO. Ob013e32832bd7e0

Instituto Nacional de Estadística y Geografía. (2018). Estudio de estadísticas sobre la disponibilidad y uso de 
las tecnologías de información y comunicación en los hogares. Recuperado de http://bit.ly/2YS2aps

Matellanes, M. (2011). Comportamientos y usos de diferentes generaciones de usuarios en Internet. Encuentros, 9(1), 107-117. Recuperado de http://bit.ly/2AzZsJt

Molero, M., Martos, A., Cardila, F., Barragán, A., Pérez-Fuentes, M., Gázquez, J., \& Rosas-Nieto, J. (2014). Uso de Internet y redes sociales en estudiantes universitarios. European Journal of child Development, Education and Psychopathology, 2(3), 81-96. http://doi. org/10.1989/ejpad.v2i3.24

Nunnally, J. C. (1978). Psychometric theory (2nd ed.). New York, NY: McGraw-Hill.

Ortiz, G., \& Gallegos, J. (2009). Acceso y usos de las tecnologías de la información y comunicación (TIC's) entre las niñas y los niños mexicanos: el caso de la Ciudad de Monterrey. Global Media Journal Edición Iberoamericana, 6(12), 71-90. Recuperado de http:// bit.ly/2BFN78w

Puerta-Cortés, D., \& Carbonell, X. (2013). Uso problemático de Internet en una muestra de estudiantes universitarios colombianos. Avances en Psicología Latinoamericana, 31(3), 620-631. http://dx.doi.org/10.12804/apl

Ramírez, M., Maldonado, G., \& Marín, V. (2015). Uso de Internet en el ámbito académico universitario. INNOEDUCA, 1(2), 69-79. Recuperado de http://bit.ly/2E4gBiM

Rial, A., Gómez, P., Braña, T., \& Varela, J. (2014). Actitudes, percepciones y uso de Internet y las redes sociales entre los adolescentes de la comunidad Gallega. Anales de Psicología, 30(2), 642-655. http://dx.doi. org/10.6018/analesps.30.2.159111
Rodrigues, R., Canales, A., Peña, T., Castro, G., \& Reyes, G. (2009). Uso de Internet del estudiantado de la Universidad Centroamericana, con enfoque en las redes sociales. Encuentro, (84), 44-61. http://dx.doi. org/10.5377/encuentro.v41i84.49

Rodríguez, C., \& Sandoval, D. (2017). Estratificación digital; acceso y usos de las TIC en la población escolar chilena. REDIE, 19(1), 20-34. https://doi.org/10.24320/ redie.2017.19.1.902

Ruiz-Olivares, R., Lucena, V., Pino, M., \& Herruzo, J. (2010). Análisis de comportamientos relacionados con el uso/abuso de Internet, teléfono móvil, compras y juegos en estudiantes universitarios. Adicciones, 22(4), 2010, 301-309. Recuperado de http://bit.ly/2RnNOIO

Sánchez, A., Oseguera, E., Corona, F., Cedeño, J., Sandoval, J., Calvillo, R., \& Cárdenas, V. (2006). Diagnostico en el uso de las TIC de los estudiantes de primer ingreso a nivel superior en la universidad de Colima. CEUPROMED, 1-11. Recuperado de http://bit.ly/2Q3QUFC

Torres-Díaz, J., Duart, J., Gómez-Alvarado, H., Marín-Gutiérrez, I., \& Segarra-Faggioni, V. (2016). Usos de Internet y éxito académico en estudiantes universitarios. Comunicar, 24(48), 61-70. https://doi. org/10.3916/C48-2016-06

Türel, Y., \& Toraman, M. (2015). The Relationship between Internet Addiction and Academic Success of Secondary School Students. Anthropologist, 20(1,2), 280288. Recuperado de http://bit.ly/2G0013u

RIDU / Revista Digital de Investigación en Docencia Universitaria / e-ISSN: 2223-2516

(C) Los autores. Este artículo es publicado por la Revista Digital de Investigación en Docencia Universitaria del Área de Institutional Research and Effectiveness de la Dirección de Aseguramiento de la Calidad, Universidad Peruana de Ciencias Aplicadas. Este es un artículo de acceso abierto, distribuido bajo los términos de la LicenciaCreativeCommons Atribución-CompartirIgual 4.0 Internacional.(http://creativecommons.org/licenses/by-sa/4.0/), que permite el uso no comercial, distribución y reproducción en cualquier medio, siempre que la obra original sea debidamente citada. 